\title{
Direct correlation between adsorption energetics and nuclear spin relaxation in liquid-saturated catalyst material
}

\author{
Neil Robinson, ${ }^{[a][b]}$ Christopher Robertson, ${ }^{[a]}$ Lynn F. Gladden, ${ }^{[a]}$ Stephen J. Jenkins ${ }^{*[b]}$ and Carmine \\ D'Agostino*[a][c]
}

\begin{abstract}
The ratio of NMR relaxation time constants $T_{1} / T_{2}$ provides ${ }_{36}$ a non-destructive indication of the relative surface affinities exhibited ${ }_{37}$ by adsorbates within liquid-saturated mesoporous catalysts. In the present work we provide supporting evidence for the existence of $a_{3}$ quantitative relationship between such measurements and adsorption energetics. As a prototypical example with relevance to green chemical processes we examine and contrast the relaxation ${ }^{4}$ characteristics of primary alcohols and cyclohexane within an industrial silica catalyst support. $T_{1} / T_{2}$ values obtained at intermediate magnetic field strength are in good agreement with $\mathrm{DFT}^{4}$ adsorption energy calculations performed on single molecules interacting with an idealised silica surface. Our results demonstrate ${ }^{4}$ the remarkable ability of this metric to quantify surface affinities ${ }^{4}$ within systems of relevance to liquid-phase heterogeneous catalysis, 48 and highlight NMR relaxation as a powerful method for the 49 determination of adsorption phenomena within mesoporous solids.
\end{abstract}

\section{Introduction}

The development of liquid-phase heterogeneous catalysis is vita for the continued utilisation of green chemical processes, such as the production of fuels and chemicals from bio-resources. ${ }^{[1-4]}$ The high molecular density present within the liquid-phase leads to the complete saturation of adsorption sites, where competitive interactions between reagents, products and solvents determine ${ }_{61}$ the relative molecular populations within the adsorbed surface layer. ${ }^{[5]}$ The ability to predict adsorption energetics based on molecular functionality ${ }^{[6-10]}$ and surface structure ${ }^{[11-14]}$ is therefore ${ }_{64}^{63}$ essential for the optimisation of selective catalytic processes ${ }_{65}$ occurring at the solid-liquid interface.

Nuclear magnetic resonance (NMR) relaxation experiments have shown particular promise in this field. Typical measurements are employed to characterise the longitudinal $\left(T_{1}\right)_{69}$ and/or transverse $\left(T_{2}\right)$ nuclear spin relaxation time constants, ${ }_{70}$ which quantify the rate at which the nuclear spin system under study regains thermal equilibrium following radiofrequency excitation with a relevant NMR pulse sequence. ${ }^{[15]}$ Specifically,

[a] Mr N. Robinson, Mr C. Robertson and Prof L. F. Gladden and Dr C D'Agostino

Department of Chemical Engineering and Biotechnology, University of Cambridge, Philippa Fawcett Drive, Cambridge, CB3 OAS, United Kingdom.

[b] Mr N. Robinson and Dr S. J. Jenkins

Department of Chemistry, University of Cambridge, Lensfield Road, Cambridge, CB2 1EW, United Kingdom.

Email: sji24@cam.ac.uk

[c] Present address:

School of Chemical Engineering and Analytical Science,

University of Manchester, Oxford Road,

Manchester, M13 9PL, United Kingdom.

Email: carmine.dagostino@manchester.ac.uk

Supporting information for this article is given via a link at the end of the document.
$T_{1}$ characterises the rate at which the sample magnetisation fully realigns with the external magnetic field, while $T_{2}$ determines the rate at which transverse phase coherence of the nuclear spin ensemble is lost. ${ }^{[16]}$ A well-established correspondence with molecular motion allows these time constants to be interpreted in terms of rotational and translational dynamics. ${ }^{[17-20]}$ Such measurements have been widely applied to the study of fluids confined to heterogeneous porous media, ${ }^{[21-24]}$ and provide information on the effects of pore structure and surface interactions on molecular dynamics. Representative fields of investigation include rock and shale wettability studies for the hydrocarbon recovery industry, ${ }^{[25-29]}$ and the characterisation of plaster and cement paste hydration kinetics. ${ }^{[30-38]}$

Recent advances have demonstrated NMR relaxation measurements as a simple and chemically selective method for comparing the surface dynamics of liquids imbibed within mesoporous catalyst materials. ${ }^{[39,40]}$ In particular, the ratio of longitudinal-to-transverse relaxation time constants $T_{1} / T_{2}$ has received considerable attention as a non-invasive indicator of the relative surface affinities exhibited by adsorbed species. This metric is typically obtained by means of a two-dimensional (2D) $T_{1}-T_{2}$ correlation pulse sequence, which, following appropriate processing in order to invert, compress and smooth the acquired NMR data in the presence of experimental noise, ${ }^{[41]}$ provides a robust method for the identification of $T_{1} / T_{2}$ values and relaxation time distributions. Weber et al. were the first to apply such analysis to liquid-saturated heterogeneous catalyst materials. ${ }^{[42]}$ In their work $T_{1} / T_{2}$ values were obtained to compare the surface affinities of solvents and reagents present during the liquid-phase hydrogenation of 2-butanone. The use of relaxation correlation measurements also allowed the timedependent displacement of these liquids to be observed when present as a mixture. Notably, it was determined that the results of these displacement experiments correlated exactly with the relative magnitude of the $T_{1} / T_{2}$ values obtained from singlecomponent experiments. The acquisition of single-component relaxation time ratios has subsequently become an established method for the prediction of competitive adsorption behaviour, and for the comparison of surface affinities within liquidsaturated catalyst materials in general. ${ }^{[43-49]}$ For example, Ralphs et al. applied 2D $T_{1}-T_{2}$ relaxation correlation experiments to compare the influence of structural modifications on the interaction of water and n-octane with a mechanochemically prepared $\mathrm{Ag} / \mathrm{Al}_{2} \mathrm{O}_{3}$ catalyst. ${ }^{[46]}$ It was observed that ball-milling of the catalyst led to an increase in the $T_{1} / T_{2}$ ratio of $n$-octane indicating an increased affinity for interactions with the surface. Conversely, the $T_{1} / T_{2}$ ratio of water was reduced, suggesting an increased propensity for the hydrocarbon to successfully compete with water for active surface sites on the ball-milled catalyst. These findings were in agreement with catalytic testing of the materials for the hydrocarbon selective catalytic reduction of $\mathrm{NO}_{\mathrm{x}}$. Further study by D'Agostino et al. expanded this investigation to include ethanol and toulene, ${ }^{[47]}$ representative of prototypical oxygenated and aromatic hydrocarbons, 
respectively. Here it was demonstrated that ethanol exhibits an ${ }_{63}$ increased capacity to displace water at the catalyst surface, ${ }_{64}$ relative to non-oxygenated hydrocarbons, facilitating greater 65 activity as a $\mathrm{NO}_{x}$ reducing agent.

The use of single-component relaxation time ratios as $a_{6}$ probe of competitive adsorption has been further investigated 68 with respect to the liquid-phase oxidation of 1,4-butanediol. ${ }^{[48]} \operatorname{In}_{69}$ this work $T_{1} / T_{2}$ ratios were obtained to observe the competitive ${ }_{70}$ adsorption of solvent and reagent molecules within a range of ${ }_{71}$ supported metal catalysts, utilising methanol as the solvent. It 72 was shown that the relative magnitude of solvent and reagent $t_{73}$ $T_{1} / T_{2}$ values provided a clear indication of catalytic performance. 74 Specifically, when $\left[T_{1} / T_{2}\right]_{\text {reagent }}>\left[T_{1} / T_{2}\right]_{\text {solvent }}$, indicating ${ }_{75}$ preferential adsorption of the reagent, the catalysts were ${ }_{76}$ observed to exhibit high activity. Conversely, for catalysts in 77 which $\left[T_{1} / T_{2}\right]_{\text {reagent }}<\left[T_{1} / T_{2}\right]_{\text {solvent }}$ a much lower activity was 78 observed, corresponding to preferential adsorption of the solvent ta $_{9}$ A clear trend was illustrated between catalytic conversion and ${ }_{80}$ the ratio of $T_{1} / T_{2}$ values obtained from reagent- and solvent- 8 saturated catalysts $\left[T_{1} / T_{2}\right]_{\text {reagent }} /\left[T_{1} / T_{2}\right]_{\text {solvent }}$, demonstrating ${ }_{82}$ not only the ability of such measurements to characterise ${ }_{83}$ competitive adsorption processes, but also the potential of ${ }_{84}$ further measurements to predict catalytic activity.

While $T_{1} / T_{2}$ measurements are now widely accepted to 86 provide valuable information concerning the relative surface affinities of liquids confined to mesoporous catalysts, the majority of such studies have been applied only as a qualitative ${ }_{87}$ indication of adsorption. As such, the observed relaxation characteristics were not considered to relate directly to the ${ }_{88}$ energetics governing adsorption processes at the solid-liquid interface. However, recent empirical developments by ${ }_{89}$ D'Agostino et al. suggest that $T_{1} / T_{2}$ values obtained at low or intermediate magnetic field strength may be directly interpreted ${ }_{91}$ as a quantitative indication of adsorption energy. ${ }^{[49]}$ This work $_{92}$ reported $T_{1}-T_{2}$ correlation experiments performed on a range ${ }_{9}$ of water-saturated mesoporous metal oxides, regularly ${ }_{94}$ employed as catalysts or catalytic supports. Through a compact ${ }_{95}$ theoretical analysis based on the surface correlation times of adsorbed water molecules it was shown that the corresponding $T_{1} / T_{2}$ ratios could be directly related to the energy of adsorption, as measured using temperature programmed desorption. Specifically, a clear relationship was shown to exist between the adsorption energy associated with the strongest adsorption sites ${ }_{101}$ on the catalyst material surface and the inverse relaxation time ratio $-T_{2} / T_{1}$; this result was rationalised on the basis that $T_{1} / T_{2103}$ values are dominated by the strongest relaxation sinks present on the pore surface.

An improved understanding of such relationships is of substantial importance with regards to furthering the application ${ }_{107}$ of nuclear spin relaxation measurements to the study of ${ }_{108}$ adsorption in liquid-saturated heterogeneous catalysis ${ }_{409}$ Accordingly, it is the purpose of the present work to validate and $\mathrm{d}_{110}$ extend our current understanding of NMR relaxation in relation to formal adsorption energetics. In this work we perform a direct comparison of NMR relaxation data with ab initio adsorption ${ }_{13}$ energy calculations, utilising periodic density functional theory ${ }_{114}$ (DFT); this approach has been selected as DFT calculations ${ }_{115}$ provide unrivalled access to the adsorption energetics of well ${ }_{116}$ defined surface interactions, and may be used to selectively $y_{117}$ probe the strongest adsorption sites available on a particular ${ }_{118}$ surface. Experimentally we investigate the relaxation properties ${ }_{119}$ of a number of small organic molecules within an industrial silica ${ }_{120}$
$\left(\mathrm{SiO}_{2}\right)$ support. Silica-based systems have previously been applied as model porous materials in a variety of the nuclear spin relaxation studies. For instance, Jonas and co-workers used a series of porous silicas to explore the influence of surface interactions and confinement effects on the relaxation characteristics of different wetting and non-wetting molecular liquids. ${ }^{[50-54]}$ Both fixed field ${ }^{[55,56]}$ and field-cycling ${ }^{[57,58]}$ relaxation measurements have been applied to the study of silicas saturated with ionic liquids (so-called ionogels), and Krzyżak et al. used $T_{1}, T_{2}$ and $T_{1}-T_{2}$ experiments to characterise and compare the relaxation behaviour of water in the ordered mesoporous silicas SBA-15 and MCM-14 at different loadings. ${ }^{[44]}$ Further, Faux et al. performed molecular dynamics simulations of water within quasi-two-dimensional nanopores bound by hydroxylated (1010) a-quartz surfaces. ${ }^{[59]}$ These simulations were used to predict the $T_{1}$ and $T_{2}$ values of confined water via calculation of the spin-pair autocorrelation function. However, to the best of our knowledge a direct comparison of NMR relaxation time ratios and DFT adsorption energy calculations has not previously been reported. As a general example of relevance to solvated green chemical processes this work investigates and compares the surface interaction strengths of a homologous series of short-chain primary alcohols and cyclohexane.

\section{Results and Discussion}

\subsection{NMR Relaxation}

$T_{1}-T_{2}$ correlations were obtained by means of a modified $2 \mathrm{D}$ NMR relaxation pulse sequence designed to minimise the influence of J-coupling interactions on our measurement of $T_{2}$ (see Experimental Section). Sections a - d of Figure 1 depict ${ }^{1} \mathrm{H}$ (proton) $T_{1}-T_{2}$ correlation plots for the alcohol-saturated silica samples investigated here; the observed correlation peaks correspond to the proton relaxation behaviour of the imbibed liquids within the silica pore structure. The observation of proton relaxation is particularly advantageous in comparison to other observable nuclei given the large number of hydrogen atoms present within typical organic molecules and the favourable NMR characteristics of the ${ }^{1} \mathrm{H}$ nucleus. The $2 \mathrm{D}$ distribution of each correlation peak represents the relative probability of the system exhibiting a given combination of $T_{1}$ and $T_{2}$ relaxation time constants, however, the correlation peak shape is also influenced by the mathematics required to invert the acquired relaxation data, which is highly susceptible to noise fluctuations. ${ }^{[41]}$ Here we concentrate on the modal relaxation times characterised by these correlation plots, and make no attempt to analyse differences in peak shapes between samples.

Figure 1a shows the 2D correlation plot for methanolsaturated silica. Two distinct correlation peaks are clearly observed, exhibiting narrow relaxation time distributions. We have previously identified multiple proton relaxation environments when investigating the $T_{1}$ relaxation of methanol within a range of mesoporous oxides. ${ }^{[60]}$ In particular, it was observed that methanol hydroxyl protons experience rapid longitudinal nuclear spin relaxation relative to those of the methyl group, leading to shorter $T_{1}$ times. Within Figure 1a we therefore assign the peak at short $T_{1}(\sim 0.15 \mathrm{~s})$ to the relaxation of protons within the methanol hydroxyl group, while the peak at longer $T_{1}(\sim 0.56 \mathrm{~s})$ is assigned to the methyl environment of the 

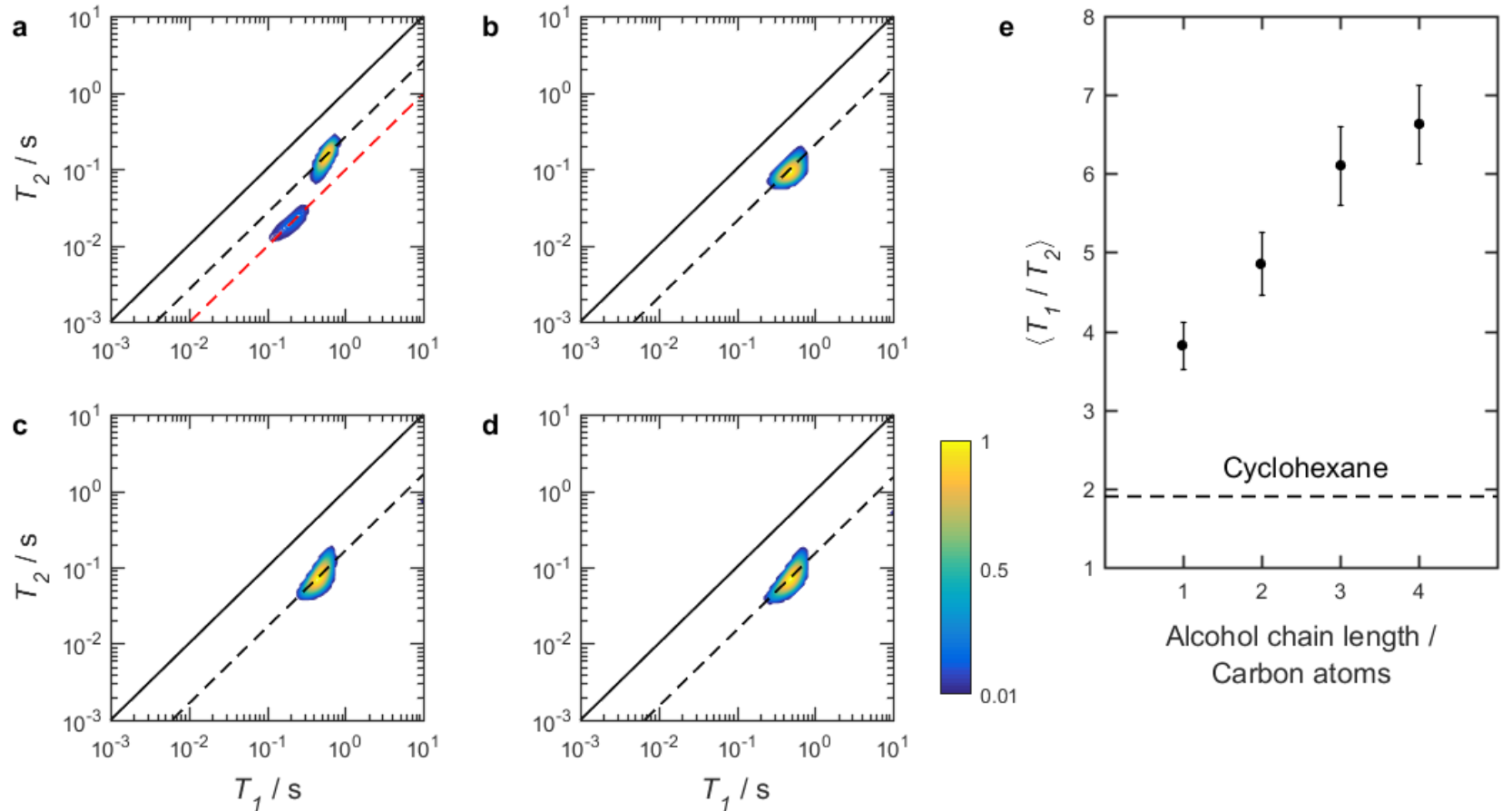

Figure 1. ' $\mathrm{H} T_{1}-T_{2}$ relaxation correlation plots of (a) methanol, (b) ethanol, (c) 1-propanol and (d) 1-butanol in mesoporous silica, acquired at $85 \mathrm{MHz}$. Solid diagonal lines indicate the parity ratio $T_{1}=T_{2}$. The observed $\left\langle T_{1} / T_{2}\right\rangle$ values are $3.8 \pm 0.3,4.9 \pm 0.4,6.1 \pm 0.5$ and $6.6 \pm 0.5$ for the alkyl environments of methanol, ethanol, 1-propanol and 1-butanol, respectively, indicated by the black dashed lines on each correlation plot. The red dashed line in (a) indicates the $\left\langle T_{1} / T_{2}\right\rangle$ ratio for the hydroxyl group of methanol, with $\left\langle T_{1} / T_{2}\right\rangle=10.4 \pm 0.8$. Alkyl group relaxation ratios are presented as a function of alcohol carbon chain length in (e), where error bar magnitudes have been determined through multiple repeat experiments. Here, the $\left\langle T_{1} / T_{2}\right\rangle$ value for cyclohexane is indicated by the dashed line, with $\left\langle T_{1} / T_{2}\right\rangle=1.9 \pm 0.1$, and represents a weakly-interacting reference measurement.

same molecules. This assignment is supported by the relative ${ }_{38}$ intensities of the two peaks, which we expect to approximately ${ }_{39}$ correlate with the number of protons within each environment, ${ }^{[42]}{ }_{40}$ weighted by any signal loss due to rapid $T_{2}$ relaxation at the pore ${ }_{4}$ surface.

A single correlation peak is observed in Figures $1 \mathrm{~b}-\mathrm{d}$, as is ${ }_{43}$ often the case for molecules imbibed in mesoporous catalyst ${ }_{44}$ materials. ${ }^{[42,43,45,46]}$ We assign these peaks to the alkyl ${ }_{45}$ environment of each alcohol; the lack of hydroxyl signal within ${ }_{46}$ these correlation plots is attributed to dominance of the acquired ${ }_{47}$ relaxation data by alkyl protons within these larger alcohols. 48 Indeed, a single correlation peak has previously been observed ${ }_{49}$ for 2-propanol imbibed within $\mathrm{Ru} / \mathrm{SiO}_{2}$ and $\mathrm{Pd} / \mathrm{Al}_{2} \mathrm{O}_{3}$ catalysts ${ }_{5}$ under similar experimental conditions ${ }^{[42,43]}$ Furthermore, a single ${ }_{5}$ relaxation environment corresponding to multiple alkyl protons ${ }_{52}$ has previously been observed in n-octane-saturated $\mathrm{Y}-\mathrm{Al}_{2} \mathrm{O}_{3},{ }^{[46]}{ }_{53}$ supporting the assignment of multiple alkyl proton environments ${ }_{54}$ to a single correlation peak within Figures $1 \mathrm{~b}-\mathrm{d}$.

The modal $T_{1} / T_{2}$ ratio of a correlation peak is quantified by ${ }_{56}$ the diagonal positioning of the distribution maximum, as ${ }_{57}$ indicated by the black dashed lines on each $T_{1}-T_{2}$ plot in ${ }_{58}$ Figure 1. Hereafter these modal values will be denoted as ${ }_{59}$ $\left\langle T_{1} / T_{2}\right\rangle$; the observed $\left\langle T_{1} / T_{2}\right\rangle$ values are approximately $3.8,4.9,60$ 6.1 and 6.6 for the alkyl peaks of methanol, ethanol, 1-propanol ${ }_{61}$ and 1-butanol, respectively. Individual modal $T_{1}$ and $T_{2}$ values ${ }_{62}$ are tabulated in the Supporting Information. Notably, due to the 63 high alkyl-to-hydroxyl proton number ratio within these ${ }_{64}$ molecules, we may interpret these alkyl relaxation time 65 characteristics as a measure of overall molecular dynamics. 66 These values are plotted as a function of carbon chain length in ${ }_{67}$ Figure 1e. As it is typical to interpret this ratio as an indicator of ${ }_{68}$ surface affinity, the positive correlation observed here suggests that short-chain primary alcohols exhibit a distinct increase in surface affinity with increasing carbon chain length, in agreement with adsorption energy measurements reported elsewhere. ${ }^{[61,62]}$ Cyclohexane, which is unable to hydrogen bond to hydroxyl groups at the pore surface, has also been examined. This system (see Supporting Information for correlation plot) comprises a single correlation peak with $\left\langle T_{1} / T_{2}\right\rangle \approx 1.9$, corroborating previous observations on the weakly-interacting nature of alkanes at oxide surfaces. ${ }^{[63]}$

Simple calculations based on the biphasic fast exchange model of Brownstein and Tarr ${ }^{[64]}$ confirm these $\left\langle T_{1} / T_{2}\right\rangle$ values are highly sensitive to relaxation within the adsorbed surface layer. In particular, we assert that for the liquid/silica systems explored here the observed relaxation time ratio is proportional to that within the adsorbed surface layer, such that $\left\langle T_{1} / T_{2}\right\rangle \propto$ $T_{1, \text { surf }} / T_{2, \text { surf }}$. Details of our calculations are provided in the Supporting Information. The assumption of biphasic fast exchange between an adsorbed surface layer and bulk-like liquid towards the centre of the pores is typical for small mesopores imbibed with rapidly diffusing non-viscous liquids, ${ }^{[21]}$ and is regularly applied to the study of liquid-saturated catalyst materials. ${ }^{[42,43,49,60]}$ Previous studies on liquid-saturated silicas suggest this approach is particularly valid for the study of liquids which wet the surface of hydroxylated pores, ${ }^{[52]}$ and has also been shown to be appropriate for the study of cyclohexane. ${ }^{[65]}$

It should be noted, in passing, that within Figure 1a, the $\left\langle T_{1} / T_{2}\right\rangle$ values for the two observed methanol proton environments are not equivalent. This observation might be expected from the polar-protic nature of the adsorbate under observation. In particular, the low intensity peak assigned 
a

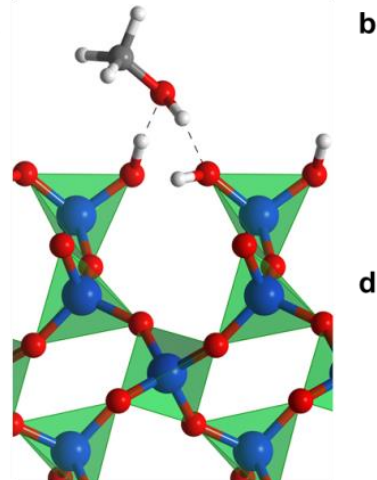

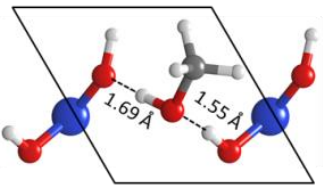

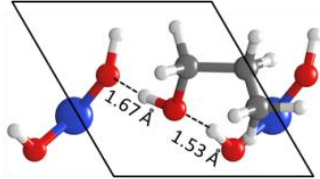

C
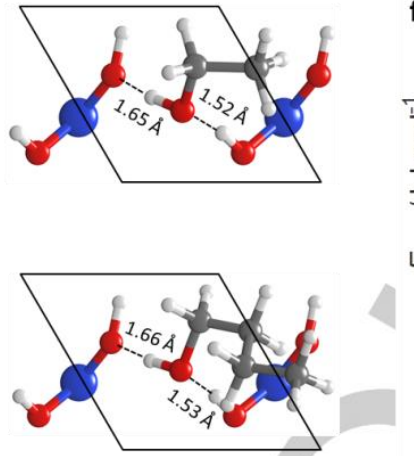

f

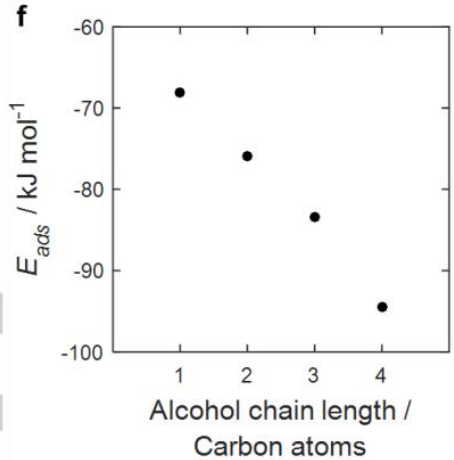

Figure 2. (a) DFT-optimised methanol adsorbed at the (0001) $\alpha$-quartz surface. Green polyhedral indicate $\mathrm{SiO}_{4}$ units within the bulk $\alpha$-quartz structure. Dashed lines indicate hydrogen bonds. (b) shows a top-down view of this same adsorbate configuration; for clarity and simplicity only surface atoms and the adsorbate are shown. (c) - (e) illustrate top-down views of (c) ethanol, (d) 1-propanol and (e) 1-butanol adsorbed at the same adsorption site. C, O, Si and $\mathrm{H}$ atoms are colourcoded grey, red, blue and white, respectively, and solid black lines indicate the $1 \times 1$ a-quartz unit cell. (f) shows the calculated adsorption energies, $E_{\text {ads }}$, of these adsorbates as a function of alcohol carbon chain length.

\begin{abstract}
to hydroxyl proton relaxation has an observed $\left\langle T_{1} / T_{2}\right\rangle$ ratio of 50 approximately 10.4 (red dashed line), which is more than double ${ }_{51}$ that of the corresponding alkyl environment $\left(\left\langle T_{1} / T_{2}\right\rangle \approx 3.8\right)$. This ${ }_{52}$ increase may be attributed to a combination of hydrogen ${ }_{53}$ bonding interactions with the pore surface - the existence of ${ }_{54}$ which will significantly hinder the motional freedom of alcohol ${ }_{55}$ hydroxyl groups relative to those within the apolar alkyl ${ }_{56}$ environment - and any proton exchange between labile hydroxyl ${ }_{57}$ protons and polar surface groups. We have recently explored ${ }_{58}$ the observation of such interactions using $T_{1}$ measurements ${ }_{59}$ elsewhere. ${ }^{[60]}$
\end{abstract}

\subsection{Ab initio adsorption energy calculations}

approach mitigates the need for a manual sampling of the surface to be performed for each adsorbate considered, and ensures binding of each alcohol at the same location. The resulting configurations for each of the adsorbed alcohols considered here are shown in Figures $2 b-e$, from which it is clear that all adsorbate molecules are bound to the surface in the same manner. Calculated adsorption energies for each of these adsorbed configurations are detailed within Figure 2f, which illustrates a near linear increase in adsorption strength with increasing carbon chain length. The adsorption of cyclohexane at our $\alpha$-quartz surface has also been investigated (see Supporting Information) and is characterised by an adsorption energy of $-31.2 \mathrm{~kJ}$ mol-1; this result confirms the weaklyinteracting nature of the alkane, relative to primary alcohols, as indicated by our relaxation experiments.

\subsection{Comparing NMR relaxation with adsorption energetics}

We now compare the acquired relaxation data with the results of our adsorption energy calculations. The aim of this comparison is to provide validation for the observation that NMR relaxation time ratios relate to the strongest adsorption site on a particular surface, and to extend our understanding of such relationships to include the characterisation of hydrocarbon adsorption. We interpret our results according to the translational surface diffusion model of Mitchell et al. ${ }^{[43]}$ As such, it is assumed that nuclear spin relaxation observed at low and intermediate field strengths occurs through the surface-adsorbate dipolar interactions of like spins, where polar adsorption sites on the catalyst support surface act as relaxation sinks. Within this formalism the ratio of relaxation time constants is given by ${ }^{[68]}$

$$
\frac{T_{1, \text { surf }}}{T_{2, \text { surf }}}=\frac{3 J(0)+5 J\left(\omega_{0}\right)+2 J\left(2 \omega_{0}\right)}{2 J\left(\omega_{0}\right)+8 J\left(2 \omega_{0}\right)},
$$

where the spectral density function $J(\omega)$ for relaxation due to surface translation is ${ }^{[43]}$

$$
J(\omega)=\tau_{m} \ln \left\{\frac{1+\omega^{2} \tau_{m}^{2}}{\left(\tau_{m} / \tau_{s}\right)^{2}+\omega^{2} \tau_{m}^{2}}\right\} .
$$

Here, $\omega_{0}$ is the Larmor frequency, and $\tau_{m}$ and $\tau_{s}$ are the translational surface correlation time and the surface residence time of the adsorbates, respectively. ${ }^{[43,49]}$ As illustrated in Figure 3, $\tau_{m}$ describes the time taken for surface hopping between adsorption sites to occur, while $\tau_{s}$ describes the average time 


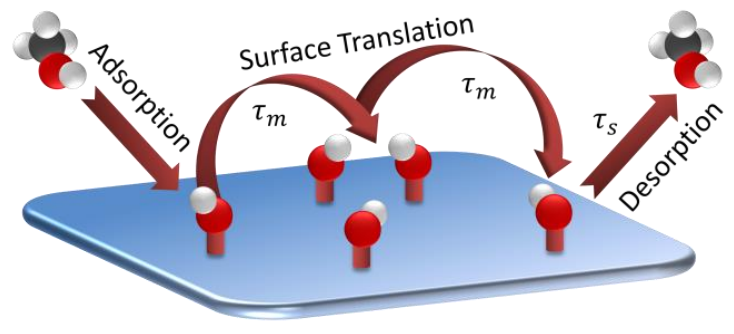

Figure 3. Illustration of the surface dynamics of alcohols across a hydroxylated pore surface, as captured by the spectral density function describing relaxation due to surface translation. Molecules first adsorb through the formation of surface-adsorbate hydrogen bonding interactions with polar surface groups. Translational motion then occurs with a characteristic correlation time $\tau_{m}$, which describes the frequency of surface hopping across the pore surface. Desorption from the surface then occurs after an average surface residence time $\tau_{s} . \mathrm{C}, \mathrm{O}$ and $\mathrm{H}$ atoms are colour-coded grey, red and white, respectively.

adsorbed molecules spend at the pore surface. ${ }^{[33,69]}$ Recalling that $\left\langle T_{1} / T_{2}\right\rangle \propto T_{1, \text { surf }} / T_{2, \text { surf }}$ Equations (1) and (2) provide $a_{52}$ clear connection between our observed relaxation ${ }_{53}$ characteristics and the adsorption phenomena captured by our ${ }^{54}$ DFT calculations.

As previously introduced, an empirical theory describing the ${ }_{57}^{56}$ relationship between these surface correlation times and $a_{58}$ formal measure of adsorption energy has recently been ${ }_{59}$ described and is of direct relevance to this work. ${ }^{[49]}$ In particular, 60 it was shown that for liquid water adsorbed within multiple ${ }_{61}$ mesoporous oxide materials the inverse ratio $-T_{2} / T_{1}$ is directly ${ }_{62}$ comparable with the desorption energy associated with the ${ }_{63}$ strongest adsorption sites present. If this theory remains robust ${ }_{64}$ for surface interactions investigated here we might expect to ${ }_{65}$ observe a linear correlation between $-1 /\left\langle T_{1} / T_{2}\right\rangle$, as obtained from our 2D $T_{1}-T_{2}$ correlation experiments, and desorption ${ }_{67}$ energy, which is readily calculated from our DFT adsorption energies as $-E_{a d s}$. This comparison is provided in Figure 4 from which an excellent agreement between our NMR relaxation ${ }_{68}$ measurements and adsorption energy calculations is clearly apparent. The dashed diagonal line is a linear fit to the acquired ${ }_{69}$ data points; notably as $E_{\text {ads }} \rightarrow 0$ this fit predicts $\left\langle T_{1} / T_{2}\right\rangle \rightarrow 1.4$, which corresponds well with the predicted value of $T_{1} / T_{2} \approx 1$ for $_{70}$ bulk liquids, ${ }^{[17]}$ and thus for totally non-interacting systems. This ${ }_{7}$ correlation provides strong evidence that the nuclear spin 72 characteristics of liquid-saturated catalyst material acquired at ${ }^{73}$ intermediate magnetic field provide a quantitative indication of ${ }^{74}$ adsorption energetics associated with the strongest adsorption ${ }^{75}$ sites present.

\section{Conclusions}

41
We have demonstrated the application of NMR relaxation time ${ }^{82}$ measurements as a non-invasive probe of surface affinity within ${ }^{83}$ systems of direct relevance to liquid-phase heterogeneous ${ }_{85}^{84}$ catalysis. Specifically, we have explored the application of $2 \mathrm{D}_{86}^{85}$ $T_{1}-T_{2}$ correlation measurements to a series of short-chain ${ }_{87}$ primary alcohols and cyclohexane within a mesoporous silica 88 support material. Through a direct comparison with ab initio ${ }^{89}$ molecular modelling we have provided clear evidence for the ${ }^{90}$ existence of a quantitative relationship between the ratio $T_{1} / T_{2}{ }^{91}$ and adsorption interactions occurring at the pore surface. $\ln _{93}^{92}$ particular, NMR relaxation time ratios have been confirmed to

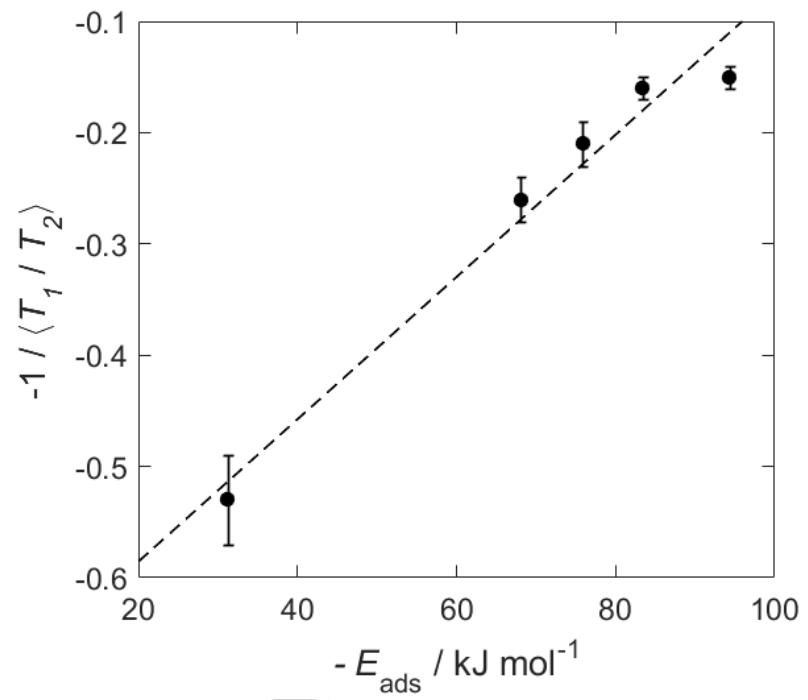

Figure 4. Comparison of the inverse NMR relaxation time ratio $-T_{2} / T_{1} \equiv-1 /\left\langle T_{1} / T_{2}\right\rangle$ with adsorption energy $E_{a d s}$ as obtained from our DFT calculations. From left to right the data points represent cyclohexane, methanol, ethanol, 1-propanol and 1-butanol. Error bars indicate the uncertainty in obtaining $\left\langle T_{1} / T_{2}\right\rangle$ for each liquid-saturated silica system, as calculated through multiple repeat experiments on multiple samples. The dashed diagonal line represents a linear fit to the data.

correlate with the strongest adsorption site present across the pore surface, providing substantial validation to previous experimental observations. The results presented here highlight the significant potential of NMR relaxation time ratios for the quantification of surface interactions within liquid-phase catalytic processes. The application of these measurements to complex pore surfaces - such as those subjected to covalent chemical modification - is the subject of further investigation.

\section{Experimental Section}

\subsection{NMR relaxation measurements}

A commercial G57 silica support (BET surface area $=272 \mathrm{~m}^{2} \mathrm{~g}^{-1}$, BJH average pore diameter $=15 \mathrm{~nm}$, BJH pore volume $=1.3 \mathrm{~cm}^{3} \mathrm{~g}^{-1}$ ) was obtained from Johnson Matthey and dried at $105^{\circ} \mathrm{C}$ for at least 12 hours before use. A pore size distribution is provided in the Supporting Information. Cyclohexane, methanol, ethanol, 1-propanol, and 1-butanol ( $\geq 99 \%$ purity, Sigma Aldrich) were used as received. Imbibed silica samples were prepared by soaking in excess liquid for at least 24 hours under ambient conditions. Samples were then separated from the liquids and rolled over a pre-soaked filter paper to remove any extrapore liquid on the outer surface of the material. Imbibed granules were transferred to sealed $7 \mathrm{ml}$ glass vials for analysis; each sample consisted of $\sim 1 \mathrm{~g}$ imbibed silica corresponding to approximately 50 granules, which ranged from 3-10 mm in diameter. As such, each experimental sample provided a well-averaged measurement of the surface-adsorbate interactions present between each molecular liquid and the pore surfaces present throughout the mesoporous silica investigated.

${ }^{1} \mathrm{H}$ relaxation measurements were performed on a Bruker Biospec horizontal bore magnet with an operational frequency of $\omega_{0} / 2 \pi=85 \mathrm{MHz}$. Sample vials were placed at the centre of a $60 \mathrm{~mm}$ birdcage coil and left for at least 15 minutes prior to analysis in order to attain thermal equilibrium. $T_{1}-T_{2}$ correlation plots were obtained by applying the 2D NMR pulse sequence shown in Figure 5 . The $T_{1}$ dimension is encoded by the application of an inversion recovery component employing $m \times \tau_{1}$ recovery delays. The $T_{2}$ dimension is encoded through $n$ refocussing loops. Here, we have replaced the CPMG echo train found in the 
standard correlation pulse sequence ${ }^{[70]}$ with a PROJECT (Periodic Refocussing of $\mathrm{J}$ Evolution by Coherence Transfer) train, ${ }^{[71]}$ comprising a series of 'perfect echoes' in order to remove any J-coupling effects on our measurement of $T_{2} \cdot{ }^{\left[{ }^{[2]}\right.}$ Echo centres are therefore separated by an ${ }^{44}$ echo time of $t_{e}=4 \tau_{2}$

In the present work, $16 \tau_{1}$ recovery delays were implemented for ${ }^{5}$ each inversion recovery, ranging between $1 \mathrm{~ms}$ and $5 \times T_{1}$ for each ${ }_{52}$ molecular liquid. The PROJECT echo train consisted of $n=512$ echoes $_{5}$ with $t_{e}=10 \mathrm{~ms}$; this value was limited by the duty cycle limitations of the ${ }_{54}$ spectrometer and by the $4 \tau_{2}$ scaling of the PROJECT echo train. The magnitude of each echo was recorded as a single data point using a oneshot technique, giving no spectral resolution. Experiments took approximately 40 minutes to complete and included 16 repeat scans to ${ }^{50}$ fulfil a full radio frequency phase cycle and provide adequate signal-to- 57 noise averaging.

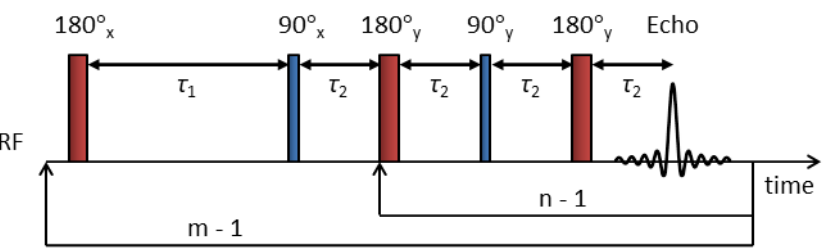

Figure 5 . The $T_{1}-T_{2}$ Inversion Recovery-PROJECT pulse sequence ${ }^{6}$ showing $180^{\circ}$ (red) and $90^{\circ}$ (blue) radio frequency (RF) pulses. $n$ refocussing ${ }^{69}$ loops lead to $n$ echoes separated by $t_{e}=4 \tau_{2}$. The sequence is repeated to ${ }^{70}$ incorporate $m \times \tau_{1}$ recovery delays.

\subsection{NMR data processing}

22 The normalised 2D NMR data acquired may be described by a Fredholm integral equation of the first kind, ${ }^{[73]}$

$$
\frac{S\left(\tau_{1}, n t_{e}\right)}{S(0,0)}=\iint_{-\infty}^{\infty} K\left(\tau_{1}, T_{1}, n t_{e}, T_{2}\right) F\left(T_{1}, T_{2}\right) d \log \left(T_{1}\right) d \log \left(T_{2}\right)+
$$

24 Here, the kernel function $K\left(\tau_{1}, T_{1}, n t_{e}, T_{2}\right)$ describes the predicted forms of 81 ${ }_{25} T_{1}$ and $T_{2}$ relaxation, ${ }^{[70]}$

$$
K\left(\tau_{1}, T_{1}, n t_{e}, T_{2}\right)=\left[1-2 \exp \left(\frac{-\tau_{1}}{T_{1}}\right)\right] \exp \left(\frac{-n t_{e}}{T_{2}}\right),
$$

26 and $\varepsilon$ represents the experimental noise, assumed to have a Gaussian ${ }^{87}$ ${ }_{27}$ distribution with zero mean. $F\left(T_{1}, T_{2}\right)$ is the desired $2 \mathrm{D}$ distribution of ${ }^{88}$ 8 relaxation time constants and was obtained via a numerical inversion of ${ }^{89}$ the acquired $2 \mathrm{D}$ data according to Equations (3) and (4). ${ }^{[70]}$ Stability of ${ }^{90}$ the inverted distribution in the presence of experimental noise was $^{9}$ achieved through the use of Tikhonov regularisation ${ }^{[74]}$ with the amplitude ${ }^{92}$ of the smoothing parameter chosen using the Generalised Cross ${ }^{93}$ Validation method. ${ }^{[41]}$

\subsection{DFT calculations}

Periodic density functional theory (DFT) calculations were performed using the plane wave code CASTEP. ${ }^{[75]}$ Structural optimisations were performed at the GGA level of theory using the PBE exchangecorrelation functional. ${ }^{[76]}$ Van der Waals interactions were accounted for by employing the semi-empirical dispersion correction by Grimme (PBED2) ${ }^{[77]}$ and all calculations made use of ultrasoft pseudopotentials to ${ }^{103}$ represent the core electrons. The plane wave basis set was expanded using a $350 \mathrm{eV}$ energy cutoff and reciprocal space was sampled using $a_{106}^{105}$ $3 \times 3 \times 1$ Monkhorst-Pack grid. ${ }^{[78]}$ Geometry optimisations were achieved through use of the BFGS minimiser, ${ }^{[79]}$ during which energies and forces ${ }_{108}^{107}$ were converged to within $2 \times 10^{-5} \mathrm{eV}$ and $0.05 \mathrm{eV} \AA^{-1}$ respectively. Further details concerning our computational approach are provided in ${ }_{110}$ the Supporting Information.

\section{Acknowledgements}

N.R. would like to thank the Catalysis@Cambridge initiative, University of Cambridge, for the award of a PhD studentship. C.R. and L.F.G. thank BP plc for the provision of a PhD studentship (C.R.). C.DA. would like to acknowledge the support of Wolfson College, Cambridge. This work made use of the Darwin Supercomputer at the University of Cambridge High Performance Computing Service.

Keywords: ab inition calculations - Adsorption $\cdot$ NMR relaxation - Porous media $\cdot$ Surface analysis

C. H. Christensen, J. Rass-Hansen, C. C. Marsden, E. Taarning, K Egeblad, ChemSusChem 2008, 1, 283-289.

[2] K. Wilson, A. F. Lee, Phil Trans $R$ Soc A 2016, 374, 20150081

[3] C. M. Friend, B. Xu, Acc. Chem. Res. 2017, 50, 517-521.

[4] A. Corma, S. Iborra, A. Velty, Chem. Rev. 2007, 107, 2411-2502

[5] C. Sievers, Y. Noda, L. Qi, E. M. Albuquerque, R. M. Rioux, S. L. Scott, ACS Catal. 2016, 6, 8286-8307.

[6] F. Abild-Pedersen, J. Greeley, F. Studt, J. Rossmeisl, T. R. Munter, P. G. Moses, E. Skúlason, T. Bligaard, J. K. Nørskov, Phys. Rev. Lett. 2007, 99, 016105

[7] J. C. F. Rodriguez-Reyes, C. G. F. Siler, W. Liu, A. Tkatchenko, C. M. Friend, R. J. Madix, J. Am. Chem. Soc. 2014, 136, 13333-13340.

C. G. F. Siler, R. J. Madix, C. M. Friend, Faraday Discuss. 2016, 188 355-368.

[9] S. Karakalos, Y. Xu, F. Cheenicode Kabeer, W. Chen, J. C. F. Rodríguez-Reyes, A. Tkatchenko, E. Kaxiras, R. J. Madix, C. M. Friend J. Am. Chem. Soc. 2016, 138, 15243-15250.

[10] Y. Xu, W. Chen, E. Kaxiras, C. M. Friend, R. J. Madix, J. Phys. Chem. B 2018, 122, 555-560.

[11] F. Calle-Vallejo, D. Loffreda, M. T. M. Koper, P. Sautet, Nat. Chem. 2015, 7, 403-410.

[12] F. Calle-Vallejo, J. Tymoczko, V. Colic, Q. H. Vu, M. D. Pohl, K Morgenstern, D. Loffreda, P. Sautet, W. Schuhmann, A. S. Bandarenka, Science 2015, 350, 185-189.

[13] A. A. Latimer, A. R. Kulkarni, H. Aljama, J. H. Montoya, J. S. Yoo, C Tsai, F. Abild-Pedersen, F. Studt, J. K. Nørskov, Nat. Mater. 2017, 16 225-229.

[14] J.-H. Zhong, X. Jin, L. Meng, X. Wang, H.-S. Su, Z.-L. Yang, C. T. Williams, B. Ren, Nat. Nanotechnol. 2017, 12, 132-136.

[15] J. Kowalewski, L. Maler, Nuclear Spin Relaxation in Liquids: Theory, Experiments, and Applications, CRC Press, 2006

[16] M. H. Levitt, Spin Dynamics: Basics of Nuclear Magnetic Resonance, Wiley-Blackwell, Chichester, England; Hoboken, NJ, 2008.

[17] N. Bloembergen, E. M. Purcell, R. V. Pound, Phys. Rev. 1948, 73, 679712 .

[18] G. Lipari, A. Szabo, J. Am. Chem. Soc. 1982, 104, 4546-4559.

[19] R. Kimmich, N. Fatkullin, Prog. Nucl. Magn. Reson. Spectrosc. 2017 $101,18-50$

[20] P. M. Singer, D. Asthagiri, W. G. Chapman, G. J. Hirasaki, J. Magn. Reson. 2017, 277, 15-24.

[21] J.-P. Korb, Prog. Nucl. Magn. Reson. Spectrosc. 2018, 104, 12-55.

[22] J.-P. Korb, New J. Phys. 2011, 13, 035016.

[23] J. Mitchell, L. F. Gladden, T. C. Chandrasekera, E. J. Fordham, Prog. Nucl. Magn. Reson. Spectrosc. 2014, 76, 1-60.

[24] J.-P. Korb, Comptes Rendus Phys. 2010, 11, 192-203.

[25] S. Godefroy, M. Fleury, F. Deflandre, J.-P. Korb, J. Phys. Chem. B 2002, 106, 11183-11190.

[26] K. E. Washburn, Concepts Magn. Reson. Part A 2014, 43A, 57-78.

[27] I. Habina, N. Radzik, T. Topór, A. T. Krzyżak, Microporous Mesoporous Mater. 2017, 252, 37-49.

[28] A. Fheed, A. Krzyżak, A. Świerczewska, J. Appl. Geophys. 2018, 151, 343-358.

[29] J.-P. Korb, B. Nicot, I. Jolivet, Microporous Mesoporous Mater. 2017 DOI 10.1016/j.micromeso.2017.05.055 
[30] K. M. Song, J. Mitchell, L. F. Gladden, J. Mater. Sci. 2009, 44, 5004-47 5012.

[31] K. M. Song, J. Mitchell, H. Jaffel, L. F. Gladden, J. Mater. Sci. 2010, 45, 5282-5290.

[32] P. J. McDonald, J. Mitchell, M. Mulheron, P. S. Aptaker, J.-P. Korb, L. 5 Monteilhet, Cem. Concr. Res. 2007, 37, 303-309.

[33] P. J. McDonald, J.-P. Korb, J. Mitchell, L. Monteilhet, Phys. Rev. E 2005, 72, 011409.

[34] L. Monteilhet, J.-P. Korb, J. Mitchell, P. J. McDonald, Phys. Rev. E5 2006, 74, 061404.

[35] H. Jaffel, J.-P. Korb, J.-P. Ndobo-Epoy, J.-P. Guicquero, V. Morin, J.57 Phys. Chem. B 2006, 110, 18401-18407.

[36] H. Jaffel, J.-P. Korb, J.-P. Ndobo-Epoy, V. Morin, J.-P. Guicquero, J. 5 Phys. Chem. B 2006, 110, 7385-7391.

[37] A. C. A. Muller, K. L. Scrivener, A. M. Gajewicz, P. J. McDonald, J.6 Phys. Chem. C 2013, 117, 403-412.

[38] F. Martini, S. Borsacchi, M. Geppi, C. Forte, L. Calucci, J. Phys. Chem. 6 C 2017, 121, 26851-26859.

[39] L. F. Gladden, J. Mitchell, New J. Phys. 2011, 13, 035001.

[40] L. F. Gladden, Curr. Opin. Chem. Eng. 2013, 2, 331-337.

[41] J. Mitchell, T. C. Chandrasekera, L. F. Gladden, Prog. Nucl. Magn. 67 Reson. Spectrosc. 2012, 62, 34-50.

[42] D. Weber, J. Mitchell, J. McGregor, L. F. Gladden, J. Phys. Chem. C6 2009, 113, 6610-6615

[43] J. Mitchell, L. M. Broche, T. C. Chandrasekera, D. J. Lurie, L. F.7 Gladden, J. Phys. Chem. C 2013, 117, 17699-17706.

[44] A. T. Krzyżak, I. Habina, Microporous Mesoporous Mater. 2016, 231, 230-239.

[45] C. D’Agostino, G. Brett, G. Divitini, C. Ducati, G. J. Hutchings, M. D. 75 Mantle, L. F. Gladden, ACS Catal. 2017, 7, 4235-4241.

[46] K. Ralphs, C. D’Agostino, R. Burch, S. Chansai, L. F. Gladden, C. 77 Hardacre, S. L. James, J. Mitchell, S. F. R. Taylor, Catal. Sci. Technol. 78 2014, 4, 531-539.

[47] C. D'Agostino, S. Chansai, I. Bush, C. Gao, M. D. Mantle, C. Hardacre, 80 S. L. James, L. F. Gladden, Catal. Sci. Technol. 2016, 6, 1661-1666.

[48] C. D'Agostino, M. R. Feaviour, G. L. Brett, J. Mitchell, A. P. E. York, G. 82 J. Hutchings, M. D. Mantle, L. F. Gladden, Catal. Sci. Technol. 2016, 6, 8 7896-7901.

[49] C. D’Agostino, J. Mitchell, M. D. Mantle, L. F. Gladden, Chem. - Eur. J.8 2014, 20, 13009-13015.

[50] G. Liu, Y. Li, J. Jonas, J. Chem. Phys. 1991, 95, 6892-6901.

J. - P. Korb, A Delville, S. Xu, G. Demeulenaere, P Costa, J. Jonas, ${ }^{88}$ Chem. Phys. 1994, 101, 7074-7081.

[53] J.-P. Korb, L. Malier, F. Cros, S. Xu, J. Jonas, Phys. Rev. Lett. 1996, 77, 91 2312-2315.
[54] S. Xu, J. Jonas, J. Phys. Chem. 1996, 100, 16242-16246.

[55] J. L. Bideau, P. Gaveau, S. Bellayer, M.-A. Néouze, A. Vioux, Phys. Chem. Chem. Phys. 2007, 9, 5419-5422.

[56] K. S. Han, X. Wang, S. Dai, E. W. Hagaman, J. Phys. Chem. C 2013 117, 15754-15762.

[57] D. Kruk, M. Wojciechowski, S. Brym, R. K. Singh, Phys. Chem. Chem Phys. 2016, 18, 23184-23194.

[58] D. Kruk, M. Wojciechowski, Y. L. Verma, S. K. Chaurasia, R. K. Singh, Phys. Chem. Chem. Phys. 2017, 19, 32605-32616.

[59] D. A. Faux, P. J. McDonald, N. C. Howlett, J. S. Bhatt, S. V. Churakov, Phys. Rev. E 2013, 87, 062309.

[60] N. Robinson, L. F. Gladden, C. D’Agostino, Faraday Discuss. 2017 204, 439-452.

[61] B. J. Stanley, G. Guiochon, Langmuir 1995, 11, 1735-1743.

[62] M. A. Natal-Santiago, J. A. Dumesic, J. Catal. 1998, 175, 252-268.

[63] C. D’Agostino, J. Mitchell, L. F. Gladden, M. D. Mantle, J. Phys. Chem. C 2012, 116, 8975-8982.

[64] K. R. Brownstein, C. E. Tarr, J. Magn. Reson. 1977, 26, 17-24.

[65] D. W. Aksnes, K. Førland, M. Stöcker, Microporous Mesoporous Mater. 2005, 77, 79-87.

[66] J. W. Han, J. N. James, D. S. Sholl, Surf. Sci. 2008, 602, 2478-2485

[67] F. Musso, M. Sodupe, M. Corno, P. Ugliengo, J. Phys. Chem. C 2009 113, 17876-17884.

[68] A. Abragam, The Principles of Nuclear Magnetism, Clarendon Press, 1961

[69] S. Godefroy, J.-P. Korb, M. Fleury, R. G. Bryant, Phys. Rev. E 2001, 64 021605.

[70] Y. Q. Song, L. Venkataramanan, M. D. Hürlimann, M. Flaum, P. Frulla, C. Straley, J. Magn. Reson. 2002, 154, 261-268.

[71] J. A. Aguilar, M. Nilsson, G. Bodenhausen, G. A. Morris, Chem Commun. 2012, 48, 811-813.

[72] K. Takegoshi, K. Ogura, K. Hikichi, J. Magn. Reson. 1969 1989, 84 611-615.

[73] L. Venkataramanan, Y. Q. Song, M. D. Hurlimann, leee Trans. Signal Process. 2002, 50, 1017-1026.

[74] A. N. Tikhonov, V. I. Arsenin, Solutions of III-Posed Problems, Winston, 1977.

[75] S. J. Clark, M. D. Segall, C. J. Pickard, P. J. Hasnip, M. I. J. Probert, K Refson, M. C. Payne, Z. Für Krist. 2005, 220, 567-570.

[76] J. P. Perdew, K. Burke, M. Ernzerhof, Phys. Rev. Lett. 1996, 77, 3865 3868

[77] S. Grimme, J. Comput. Chem. 2006, 27, 1787-1799.

H. J. Monkhorst, J. D. Pack, Phys. Rev. B 1976, 13, 5188-5192.

[79] B. G. Pfrommer, M. Côté, S. G. Louie, M. L. Cohen, J. Comput. Phys 1997, 131, 233-240. 


\section{Entry for the Table of Contents}

\section{ARTICLE}

\section{Nuclear spin relaxation}

measurements provide insight into the surface dynamics of adsorbed liquids within optically opaque mesoporous media, such as heterogeneous catalysts. We illustrate that the ratio of relaxation time constants $T_{1} / T_{2}$ - obtained for a range of short-chain primary alcohols and cyclohexane in saturated mesoporous silica provides quantitative information on molecular surface affinity, and is comparable to the results of DFT adsorption energy ( $\left.E_{\text {ads }}\right)$ calculations.

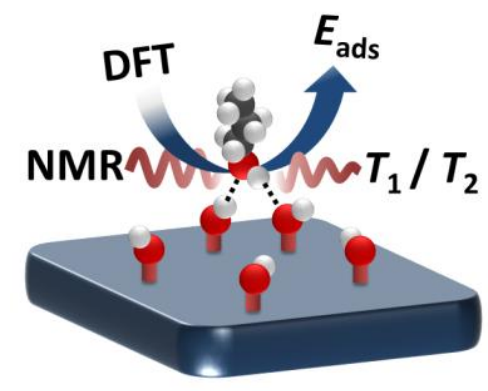

Neil Robinson. Christopher Robertson, Lynn F. Gladden, Stephen J. Jenkins* and Carmine D'Agostino*

Page No. - Page No.

Direct correlation between adsorption energetics and nuclear spin relaxation in liquid-saturated catalyst material 